\title{
А.Н. ВОРОНЕЦКИЙ
}

\section{ЭНТЕРОСКОПИЯ ПРИ БОЛЕЗНИ КРОНА У ДЕТЕЙ С ПОСЛЕДОВАТЕЛЬНЫМ ПРИМЕНЕНИЕМ РАСТВОРИМОЙ И ЭНДОВИДЕО КАПСУЛ}

\author{
УО «Белорусский государственный медицинский университет», \\ Республика Беларусь
}

Цель. Продемонстрировать эффективность применения эндовидеокапсульной энтероскопии при болезни Крона у детей с предварительным использованием растворимой капсулы для оценки проходимости кишки и контроля терапии.

Материал и методы. Использованы эндоскопический метод диагностики проходимости тонкой кишки с применением растворимой капсулы «РillCam patency» и последующая энтероскопия с применением эндовидеокапсулы «РіllCam» у детей с болезнью Крона. В статье описаны два клинических наблюдения пациентов с болезнью Крона, из которых у одного произошла длительная миграции капсулы по причине наличия стриктур тонкой кишки.

Результаты. На клинических примерах обосновано эндоскопическое исследование детей с болезнью Крона в два этапа: на первом этапе с помощью растворимой капсулы проверяется проходимость кишки, при условии сохранения проходимости кишки на втором этапе проводят энтероскопию с применением эндовидеокапсулы.

Установлено, что задержка миграции эндовидеокапсулы в процессе обследования возможна. Это не требует активного вмешательства, если у пациента нет признаков кишечной непроходимости. Однако при условии задержки миграции капсулы за пациентом необходимо динамическое наблюдение в условиях хирургического стационара. В нашем наблюдении капсула продолжала оставаться в тонкой кишке более 7 месяцев с последующей самопроизвольной элиминацией. Одним из эффективных методов диагностики задержки капсулы является выполнение обзорной рентгенограммы брюшной полости, так как элементы питания капсулы являются рентгеноконтрастными.

Заключение. Осложнением болезни Крона у детей может быть сужение просвета и проходимости тонкой кишки, поэтому перед проведением эндовидеокапсульной энтероскопии следует установить проходимость кишки. Беспрепятственная миграция растворимой капсулы является основанием для последующей капсульной эндоскопии. При задержке эндовидеокапсулы в кишке и сохранении пассажа содержимого кишки необходимо обеспечить за пациентом клиническое динамическое наблюдение до элиминации капсулы.

Ключевые слова: капсульная энтероскопия, болезнь Крона, растворимая капсула, миграция эндовидеокапсулы, пассаж, стриктура кишки, эндоскопия

Objectives. To demonstrate the effectiveness of endovideocapsule enteroscopy in children with Crohn's disease by preliminary use of a soluble capsule to assess the intestinal patency and therapy monitoring.

Methods. The endoscopic method for diagnosing of the small intestine patency with using a soluble capsule «PillCam patency» and subsequent enteroscopy using endovideocapsule «PillCam» in children with Crohn's disease had been applied. This article describes two clinical observations of patients with Crohn's disease; a continuous migration of the capsule due to the presence of strictures of the small intestine took place in one of those patients.

Results. The two-stage endoscopic examination of children with Crohn's disease is justified using clinical examples: at the first stage the intestinal patency is checked using a soluble capsule, if the intestine is passable, at the second stage enteroscopy using endovideocapsule is carried out.

The delay of endovideocapsule migration during the examination has been established to be possible. It does not require an active intervention if a patient has no signs of intestinal obstruction. However, in case of the capsule migration delay, the patient must be dynamically observed in the surgical hospital. In our observation the capsule remained in the small intestine over 7 months with subsequent spontaneous elimination. One of the effective diagnostic methods of capsule delay is the performance of a plain abdominal X-ray, since the capsule batteries are radiopaque.

Conclusion. One of Crohn's disease complications in children can be a narrowing of the small intestine lumen and its patency, so prior the endovideocapsule enteroscopy the intestinal patency should be checked. The unimpeded migration of a soluble capsule is thought to be a basis for the subsequent capsule endoscopy. In case of endovideocapsule delay in the intestine and preservation passage of the intestinal contents it is necessary to ensure patient's clinical dynamic observation until the elimination of the capsule.

Keywords: capsule enteroscopy, Crohn's disease, soluble capsule, migration of a soluble capsule, passage, intestinal stricture, endoscopy 
Novosti Khirurgii. 2017 Jan-Feb; Vol 25 (1): 99-105

Enteroscopy in Children with Crohn's Disease wiht Application of Soluble and Endovideo Capsules A.N. Voronetsky

\section{Введение}

Болезнь Крона (БК) у детей ввиду ее длительного хронического рецидивирующего течения и возникающих осложнений находится в фокусе внимания многих врачей-специалистов: педиатров, гастроэнтерологов, детских хирургов, эндоскопистов. Данные о распространенности БК неоднозначны и зависят от региона исследования, использованных методов диагностики, систематизации учета. В систематическом обзоре, базируюшемся на 8103 источниках литературы (1950-2010 гг.), 4975 цитатах (1980-2010 гг.) и на результатах 167 исследований в Европе (19302008), 52 исследований в Азии и на Ближнем Востоке (1950-2008) и 27 исследований в Северной Америке (1920-2004) приводятся данные о частоте болезни Крона в разных регионах. Из расчета на 100000 человеко-лет получены данные о числе заболевших: 12,7 в Европе, 5,0 в Азии и на Ближнем Востоке, 20,2 в Северной Америке. Показатель распространенности болезни Крона имеет максимальные значения в Европе (322 на 100000 человек) и Северной Америке (319 на 100000 человек) со статистически значимой тенденцией к росту по всему миру, что позволяет говорить о болезни Крона как о глобальном заболевании [1]. В обзоре Д.С. Ставцева сообщается, что в России согласно статистическим данным показатели распространенности БК ниже среднеевропейских и составляют от 1-1,2 до 3-4 случаев на 100000 населения, что связывают с недостаточным учетом пациентов и низкой распознаваемостью болезни, когда ее диагностика занимает 3-6 лет и более [2].

Диагноз БК выставляется на основании сочетания данных анамнеза, клиники, результатов лабораторного, рентгенологического, эндоскопического, гистологического исследований [3]. В ряде случаев для дифференциальной диагностики требуется ультразвуковое исследование, компьютерная и магнитно-резонансная томография. Критерии диагноза БК по основным признакам сформулировал Lennard-Jones [4, 5]. Дифференциальная диагностика БК необходима с многогранной патологией, язвенный колит, острые кишечные инфекции, глистные инвазии, паразитозы, антибиотико-ассоциированные состояния, туберкулез кишки, системный васкулит, неоплазия кишки, дивертикулит, аппендицит, эндометриоз, солитарная язва прямой кишки, ишемический колит, актиномикоз, лучевые поражения кишки, синдром раздраженного кишечника, но основным ее симптомом которой является хроническая диарея $[6,7,8,9]$.

Приведенные данные о распространенности БК, особенностях диагностики относятся ко взрослым пациентам, но известно, что болезнью Крона болеют в любом возрасте, в том числе дети. Сведения об эффективной диагностике БК, контроле течения болезни, результативности лечения у детей в отечественной литературе отсутствуют, а в зарубежной единичны [10, 11, 12]. В диагностике предпочтение отдается информативным, безопасным, экономным методам, из которых в настоящее время приоритетны методы визуализации, в частности эндоскопия.

Цель. Продемонстрировать эффективность применения эндовидеокапсульной энтероскопии при болезни Крона у детей с предварительным использованием растворимой капсулы для оценки проходимости кишки и контроля терапии.

\section{Материал и методы}

Впервые в Беларуси эндоскопический метод диагностики проходимости тонкой кишки с применением растворимой капсулы и последующей энтероскопией с применением эндовидеокапсулы у детей с болезнью Крона был использован нами на базе УЗ «Городская клиническая больница № 1» г. Минска в 2013 году [13, 14]. За истекший период совместно сотрудниками эндоскопического отделения и кафедры детской хирургии УО «Белорусский государственный медицинский университет» проанализированы результаты исследований, сформулированы показания и противопоказания для применения данного метода у детей.

В настоящей статье приводятся клинические примеры обследования детей с длительно текущей болезнью Крона средней и высокой степени активности. Возраст пациентов 10 и 16 лет. Выбор демонстрируемых клинических случаев обусловлен различной избранной тактикой обследования детей, когда в одном случае было принято решение об использовании капсульной эндовидеоэнтероскопии у ребенка с суженным просветом тонкой кишки и длительной задержкой капсулы в кишке, а в другом случае обоснованно применили предварительную диагностику проходимости тонкой кишки с использованием растворимой капсулы и после полученного положительного результата провели эндовидеоэнтероскопию. Дети находились на обследовании и лечении 
в гастроэнтерологическом отделении УЗ «3-я Детская городская клиническая больница». Диагноз был подтвержден лабораторными и инструментальными методами. Капсульная энтероскопия была показана для определения распространенности патологического процесса и характера изменения слизистой оболочки кишки. Учитывая степень активности болезни Крона и высокий риск формирования стриктур кишки, детям выполнялись диагностические мероприятия в два этапа. Первым этапом оценивали проходимость кишки с применением системы «PillCam patency» («Given Imaging», Израиль), состоящей из растворимой капсулы и сканера для обнаружения капсулы в кишке.

Система «PillCam patency» предназначена для применения у пациентов с высоким риском стриктур или обструкции кишки при воспалительных заболеваниях кишечника, при подозрении опухоли кишки и др. Оболочка растворимой капсулы состоит из лактозы, что обеспечивает биологическую деградацию в просвете кишки, а также имеет примесь бария для рентгенконтрастности капсулы. Наружная поверхность растворимой капсулы непроницаема для жидкости, но в торцевой части капсулы имеется окошко, через которое содержимое тонкой кишки медленно проникает внутрь капсулы, и под действием ферментов оболочка капсулы медленно разрушается. Внутри растворимой капсулы находится миниатюрный датчик размером $3,0 \times 13,0$ мм, передающий радиочастотные сигналы, а после разрушения оболочки капсулы радиодатчик способен мигрировать по кишке даже с очень узким просветом. После проглатывания капсулы проводят сканирование для ее обнаружения. Деградация капсулы может продолжаться до 80-100 часов после ее проглатывания, но начинает разрушаться капсула спустя 30 часов. Наружные размеры растворимой капсулы идентичны размерам применяемой нами эндовидеокапсулы и составляют соответственно $11,0 \times 26,0$ мм и $11 \times 26 \times 31$ мм. Если через 30 часов растворимая капсула в кишке не определяется, это означает, что кишка проходима и возможно проведение второго этапа обследования - энтероскопии с использованием эндовидеокапсулы.

Для проведения капсульной энтероскопии у детей использована эндовидеокапсула модели «PillCam». Капсула одноразовая, она изготовлена из биологически неактивных материалов, безопасна для употребления внутрь, не вызывает затруднений при проглатывании. При миграции по желудочно-кишечному тракту видеокапсула не вызывает субъективных ощущений у пациента, из кишечника выводится естественным путем.
В состав капсулы входят четыре светодиода, линза, микросхема цветной камеры, две батареи, радиочастотный передатчик и антенна. Камера создана на основе CMOS-матрицы (CMOS complementary metal oxide semiconductor комплементарный металло-оксидный полупроводник). Этому типу матрицы требуется значительно меньше мощности по сравнению с CCD-матрицей в приборах с зарядовой связью, встроенной в видеоэндоскопы и цифровые камеры, поэтому она может работать при очень низком уровне освещения. Капсула регистрирует изображение тонкой кишки с частотой два кадра в секунду и передает данные по радиочастотам на записывающее устройство, находящееся на поясе пациента. Как только запись завершена, данные с записывающего устройства обрабатываются на компьютерной рабочей станции, программное обеспечение которой позволяет провести полный анализ полученного изображения на мониторе компьютера. В среднем в течение 8-часового исследования записывается до 50000 кадров.

\section{Результаты}

Приводим два клинических примера обследования детей 12 и 16 лет с болезнью Крона. В первом клиническом случае была проведена капсульная энтероскопия без предварительного применения растворимой капсулы и оценки проходимости кишки, у другого пациента последовательно использованы растворимая капсула с последующей капсульной энтероскопией. Оба ребенка были направлены из гастроэнтерологического отделения УЗ «3-я Детская городская клиническая больница» в эндоскопическое отделение УЗ «Городская клиническая больница № 1» г. Минска для обследования.

Пациентка, 12 лет, находится на диспансерном учете в детской поликлинике по месту жительства, в 2012 году диагностирована болезнь Крона, в 2013 году - целиакия. Диагноз основной: «Болезнь Крона, тонкокишечная форма, осложненная стенозом тонкой кишки, тяжелой степени, непрерывно рецидивирующее течение, гормонозависимая форма, активность 2 степени. Код К 50». Сопутствующее заболевание: «Целиакия. Низкорослость смешанного генеза. Хроническая белково-энергетическая недостаточность 2 степени. Вторичный остеопороз. Дисбиоз кишечника 1 степени, стадия компенсации». 08.04.2014 г. пациентке выполнена капсульная энтероскопия. Исследование тонкой кишки продолжалось 13 часов - максимальный промежуток времени, на который рассчитана мощность батареи, в течение этого времени проводилась регистрация видеоизображения слизистой тон- 


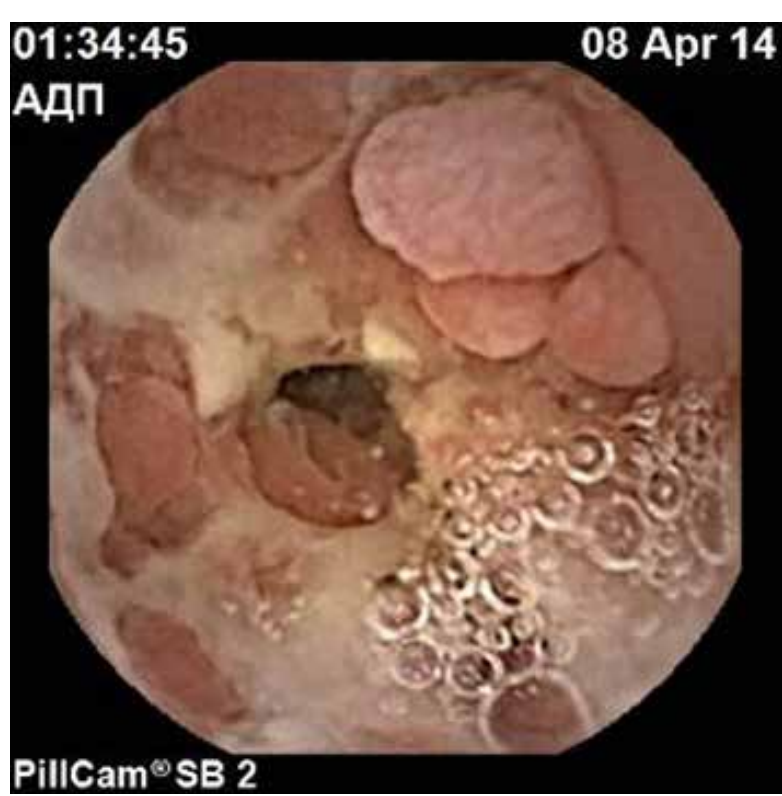

Рис. 1. Пациентка 12 лет. 2\% прохода капсулы, 2\% времени. Сливающиеся язвы с псевдополипами тощей кишки

кой кишки. В момент окончания исследования капсула находилась в дистальном отрезке тонкой кишки, естественной элиминации капсулы из кишечника не произошло. Заключение капсульной эндоскопии: «На осмотренных участках тонкой кишки определяются множественные язвы неправильной формы, псевдополипы до 1,2 см в диаметре, множественные геморрагические эрозии. Слизистая оболочка тонкой кишки отечная, рыхлая, с очаговым налетом фибрина. На отдельных участках просвет кишки сужен. Имеются эндоскопические признаки болезни Крона»

Рис. 3. Пациентка 12 лет. 99\% прохода капсулы, 94\% времени. Villous oedema

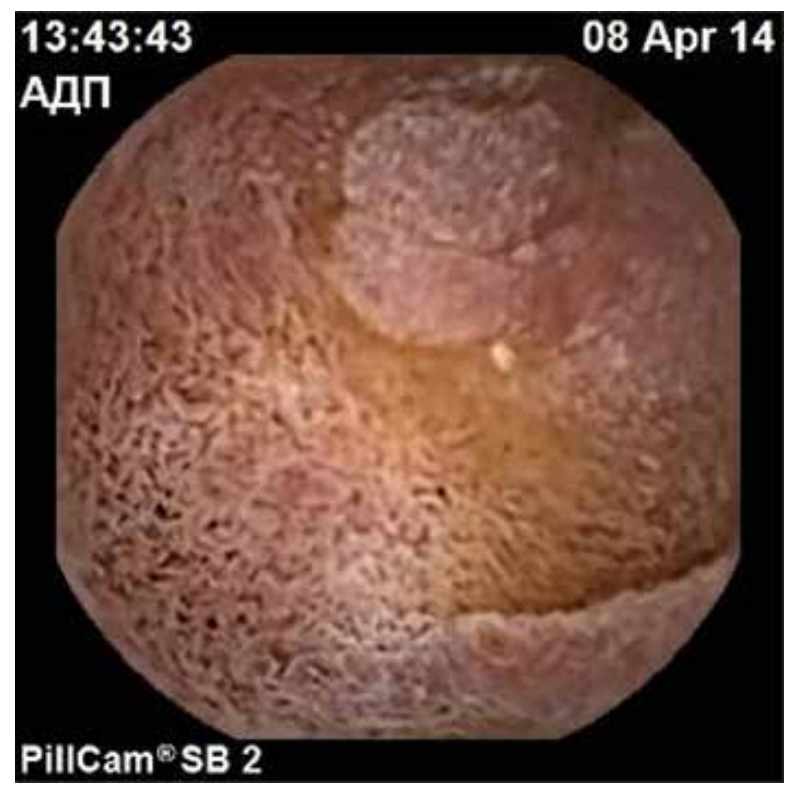

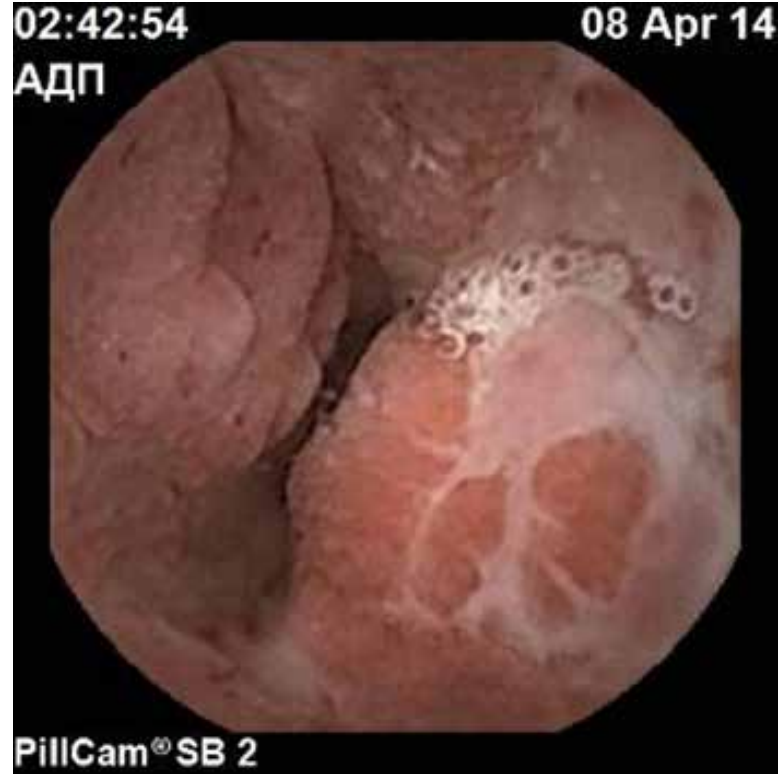

Рис. 2. Пациентка 12 лет. 4\% прохода капсулы, $11 \%$ времени. Деформация (сужение) просвета, язва, геморрагические эрозии

(рис. 1, 2, 3, 4).

09.04.2014 г. выполнена обзорная рентгенограмма брюшной полости, на которой подтверждено наличие эндовидеокапсулы в тонкой кишке (рис. 4). Поскольку капсула не нарушала пассаж содержимого по тонкой кишке, рекомендовано динамическое наблюдение за миграцией капсулы по кишке, при возникновении клиники кишечной непроходимости - госпитализация. В последующем многократно в период с сентября 2014 г. по март 2015 г. пациентка находилась под наблюдением, производились плановые

Рис. 4. Пациентк 12 лет. Обзорная рентгенограмма брюшной полости. В подвздошной области контрастируются батареи эндовидеокапсулы

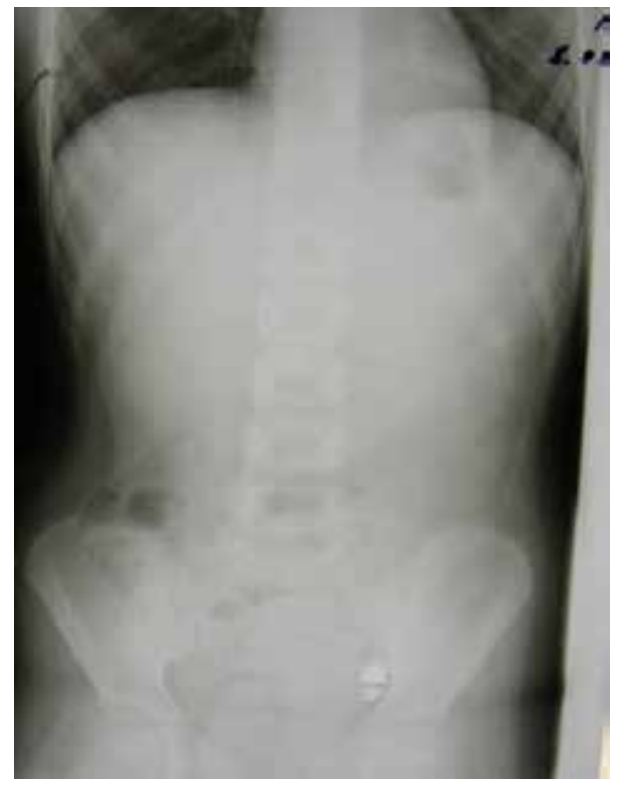


госпитализации в УЗ «Могилевская детская областная больница», Республиканский детский центр целиакии на базе УЗ «3-я Детская городская клиническая больница» с целью проведения антицитокиновой терапии при продолжающейся базовой терапии. Диагноз основной тот же, в сопутствующем диагнозе: «Инородное тело кишечника (эндоскопическая капсула после энтероскопии от 08.04.2014 г.)». При госпитализации в период 29.12.2014-01.01.2015 инородное тело в тонкой кишке в виде эндоскопической капсулы продолжало определяться, а в последующей госпитализации при рентгенографии брюшной полости от 10.02.2015 г. капсула не визуализирована и в последующем при ультразвуковом и рентгенологическом исследовании не определялась.

Пациент, 16 лет. Диагноз основной: «Болезнь Крона, тонко-толстокишечная форма, непрерывно рецидивирующее течение, средней степени. Код К 50.0». Сопутствующее заболевание: «Перианальный свищ. Хронический атрофический активный бульбит. Недостаточность кардии, дуодено-гастральный рефлюкс. Хронический антральный гастрит, умеренно выраженный, слабоактивный, с признаками атрофии, хронический фундальный гастрит слабоактивный. Бронхиальная астма, аллергическая, ремиссия». Показанием для обследования тонкой кишки явилась оценка степени протяженности и активности болезни Крона. Назначено исследование проходимости тонкой кишки с применением растворимой капсулы «PillCam patency». 07.04.2016 ребенок самостоятельно проглотил капсулу, после чего регистрировали нормальную миграцию растворимой капсулы по

Рис. 5. Пациент 16 лет. 45\% прохода капсулы, $38 \%$ времени. Язва дистальных отделов тощей кишки

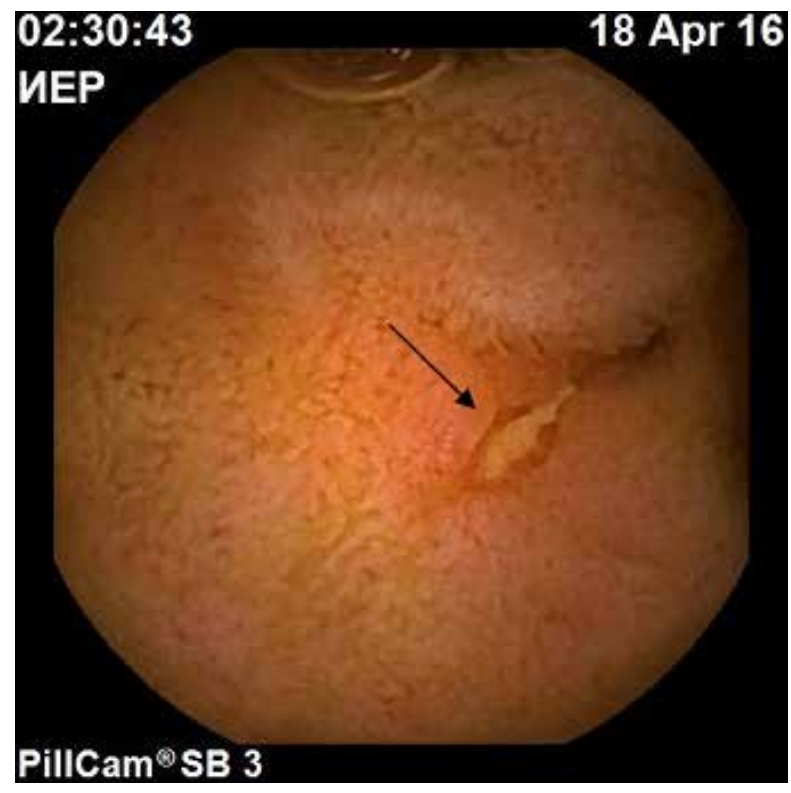

кишечнику в течение 30 часов и ее естественную элиминацию. Учитывая нормальную скорость миграции капсулы «PillCam patency», что указывало на сохраненную проходимость кишки и отсутствие противопоказаний для капсульной энтероскопии, 18.04.2016 выполнена энтероскопия с использованием эндовидеокапсулы «PillCam». Время прохождения видеокапсулой тонкой кишки составило 4 ч. 39 мин. При анализе видеоэндоскопических данных тонкой кишки отмечаются эрозии и язвы на всем ее протяжении (рис. 5,6 ). На фоне нормальной и воспалительно измененной слизистой оболочки тонкой кишки определяются афтозные поражения и щелевидные язвы, рельеф слизистой оболочки терминального отдела подвздошной кишки имеет вид «булыжной мостовой». В тощей кишке визуализируется непродолжительный сегмент рассеянной лимфангиэктазии. В проксимальном отделе подвздошной кишки определяется полип и псевдополип у края язвы. Указанные видеоэндоскопические признаки соответствуют картине болезни Крона тонкой кишки.

\section{Обсуждение}

Описанный первый клинический пример пациентки 12 лет демонстрирует следующее:

- тонкокишечная форма болезни Крона тяжелой степени с рецидивирующим течением может быть осложнена стенозом тонкой кишки, препятствующим миграции эндовидеокапсулы по кишке;

- задержка эндовидеокапсулы в тонкой кишке не препятствует пассажу содержимого

Рис. 6. Пациент 16 лет. 98\% прохода капсулы, 93\% времени. Щелевидная язва, стенка по типу «бульжной мостовой» терминального отдела подвздошной кишки

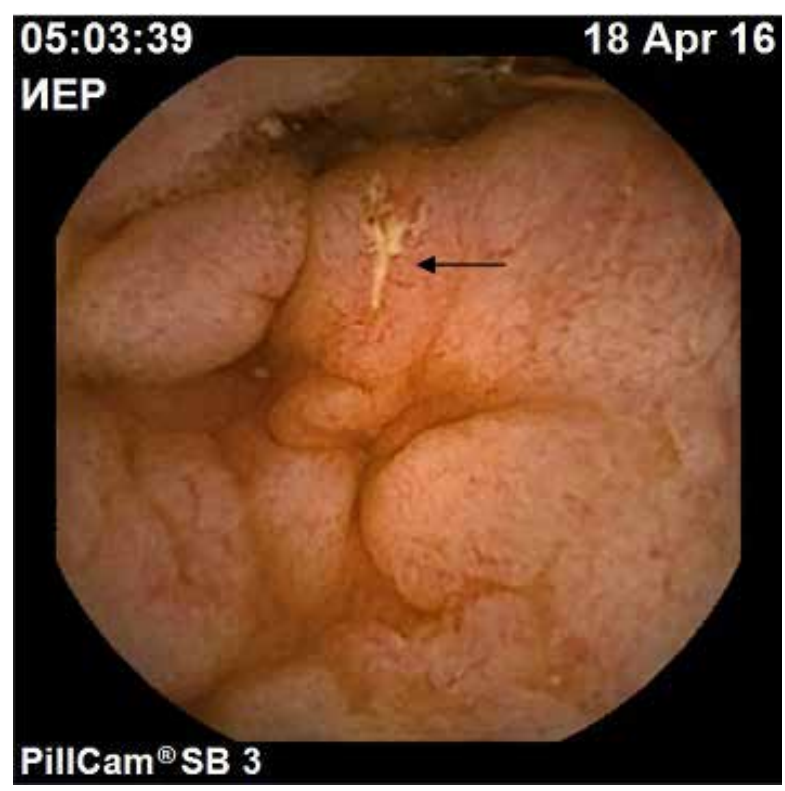


по кишке и не вызывает кишечную непроходимость;

- длительность нахождения эндовидеокапсулы в тонкой кишке в нашем наблюдении составила восемь месяцев с последующей самопроизвольной элиминацией;

- при длительной задержке эндовидеокапсулы в тонкой кишке требуется динамическое наблюдение за состоянием пациента и миграцией капсулы с применением ультразвукового и рентгенологического методов исследования;

- эффективная базовая терапия болезни Крона при условии улучшения состояния слизистой оболочки кишки и пассажа кишечного содержимого способствует естественной элиминации эндовидеокапсулы;

- задержка эндовидеокапсулы в желудочно-кишечном тракте в отдельных случаях не требует инструментальных вмешательств для извлечения капсулы.

На клиническом примере пациента 16 лет с болезнью Крона показано поражение тонкой кишки на всем ее протяжении, что имеет высокий риск стриктур, сужения просвета кишки. Поэтому для оценки проходимости кишки перед проведением эндовидеоэнтероскопии было выполнено исследование с применением растворимой капсулы.

Визуализацию слизистой оболочки кишки обеспечивает эндоскопия, когда можно выявить признаки, характерные для БК: прерывистое поражение слизистой оболочки, глубокие продольные и поперечные язвы с островками отечной гиперемированной слизистой оболочкой (симптом «булыжной мостовой»), линейные язвы в виде трещины, афты, стриктуры, устья свищей.

\section{Выводы}

1. Осложнением болезни Крона у детей может быть сужение просвета и проходимости тонкой кишки, поэтому перед проведением эндовидеокапсульной энтероскопии следует установить проходимость кишки.

2. Использование системы «PillCam patency» у детей с болезнью Крона позволяет безболезненно и безвредно установить стеноз или обструкцию тонкой кишки, что относится к противопоказаниям для проведения капсульной энтероскопии. При сужении просвета кишки регистрируется задержка миграции растворимой капсулы, оболочка которой, благодаря своей структуре, под влиянием пищеварительных ферментов подвергается деградации, а миниатюрный радиодатчик элиминируется естественным путем.

3. Беспрепятственная миграция раство- римой капсулы является основанием для последующей капсульной эндоскопии.

4. При задержке эндовидеокапсулы в кишке следует детально изучить видеозапись, сделанную камерой капсулы, установить локализацию капсулы и, по возможности, причину ее задержки. Если при этом сохранен пассаж содержимого кишки, необходимо обеспечить за пациентом клиническое динамическое наблюдение до элиминации капсулы.

\section{Клинические случаи демонстрируются с согласия родителей. Конфликт интересов отсутствует. \\ Работа выполнялась в соответствии с планом научных исследований УО «Белорусский государственный медицинский университет». Финансовой поддержки со стороны компаний- производителей автор не получал.}

\section{ЛИТЕРАТУРА}

1. Molodecky NA, Soon IS, Rabi DM, Ghali WA, Ferris M, Chernoff $G$, et al. Increasing incidence and prevalence of the inflammatory bowel diseases with time, based on systematic review. Gastroenterology. 2012 Jan;142(1):46-54.e42; quiz e30. doi: 10.1053/j. gastro.2011.10.001.

2. Ставцев ДС, Астрелина ТА, Князев ОВ, Пухликова ТВ. Значение иммуногенетических факторов в развитии болезни Крона. Рос Журн Гастроэнтерологии Гепатологии Колопроктологии. 2015;(3):70-77. 3. Monteiro S, de Castro JD, Carvalho PB, Moreira MJ, Rosa B, Cotter J. PillCam ${ }^{\circledR}$ SB3 capsule: Does the increased frame rate eliminate the risk of missing lesions? World J Gastroenterol. 2016 Mar 14; 22(10): 3066-68. doi: 10.3748/wjg.v22.i10.3066.

4. Lennard-Jones JE. Classification of inflammatory bowel disease. Scand J Gastroenterol. 1989;170(Suppl):2-6; discussion 16-9.

5. Boucher AL, Pereira B, Decousus S, Goutte M, Goutorbe F, Dubois A, et al. Endoscopy-based management decreases the risk of postoperative recurrences in Crohn's disease. World J Gastroenterol. 2016 Jun 7;22(21):5068-78. doi: 10.3748/wjg.v22.i21.5068.

6. American Gastroenterological Association medical position statement: Guidelines for the evaluation and management of chronic diarrhea. Gastroenterology. 1999 Jun;116;(Is 6):1461-63. doi: http://dx.doi. org/10.1016/S0016-5085(99)70512-3.

7. Проект клинических рекомендаций по диагностике и лечению взрослых пациентов с болезнью Крона. Колопроктология. 2013;(3 Прил):22-38.

8. Santos-Antunes J, Cardoso H, Lopes S, Marques M, Nunes AC, Macedo G. Capsule enteroscopy is useful for the therapeutic management of Crohn's disease. World J Gastroenterol. 2015 Nov 28;21(44):12660-66. doi: 10.3748/wjg.v21.i44.12660.

9. Kilcoyne A, Kaplan JL, Gee MS. Inflammatory bowel disease imaging: Current practice and future directions. World J Gastroenterol. 2016 Jan 21;22(3):91732. doi: $10.3748 /$ wjg.v22.i3.917.

10. Bennett JL, Ha CY, Efron JE, Gearhart SL, Lazarev MG, Wick EC. Optimizing perioperative Crohn's disease management: role of coordinated medi- 
cal and surgical care. World J Gastroenterol. 2015 Jan 28;21(4):1182-88. doi: 10.3748/wjg.v21.i4.1182.

11. Bettenworth D, Nowacki TM, Cordes F, Buerke B, Lenze F. Assessment of stricturing Crohn's disease: Current clinical practice and future avenues. World $J$ Gastroenterol. 2016 Jan 21;22(3):1008-16. doi: 10.3748/ wjg.v22.i3.1008.

12. Podugu A, Tandon K, Castro FJ. Crohn's disease presenting as acute gastrointestinal hemorrhage. World J Gastroenterol. 2016 Apr 28;22(16):4073-78. doi: 10.3748/wjg.v22.i16.4073

13. Воронецкий АН, Лях ОМ, Дергачев АВ. Применение эндовидеокапсулы для диагностики патологии тонкой кишки у детей. Здравоохранение. 2015 Май;(5):32-34.

14. Воронецкий АН, Лях ОМ, Лесковская СВ, Саванович ИИ, Дергачев АВ. Применение растворимой капсулы для диагностики проходимости тонкой кишки у детей. Здравоохранение. 2016;(4):28-32.

\section{REFERENCES}

1. Molodecky NA1, Soon IS, Rabi DM, Ghali WA, Ferris M, Chernoff G, Benchimol EI, Panaccione R, Ghosh S, Barkema HW, Kaplan GG. Increasing incidence and prevalence of the inflammatory bowel diseases with time, based on systematic review. Gastroenterology. 2012 Jan;142(1):46-54.e42; quiz e30. doi: 10.1053/j.gastro.2011.10.001. Epub 2011 Oct 14.

2. Stavtsev DS, Astrelina TA, Kniazev OV, Pukhlikova TV. Znachenie immunogeneticheskikh faktorov $\mathrm{v}$ razvitii bolezni Krona [Value of immunogenetic factors in the development of Crohn's disease] Ros Zhurn Gastroenterol, Gepatol, Koloproktol. 2015;(3):70-77. 3. Monteiro S, de Castro JD, Carvalho PB, Moreira MJ, Rosa B, Cotter J. PillCam ${ }^{\circledR}$ SB3 capsule: Does the increased frame rate eliminate the risk of missing lesions? World J Gastroenterol. 2016 Mar 14; 22(10): 3066-68. doi: 10.3748/wjg.v22.i10.3066.

Published online 2016 Mar 14. doi: 10.3748/wjg.v22. i10.3066 PMCID: PMC4779931

4. Lennard-Jones JE. Classification of inflammatory bowel disease. Scand J Gastroenterol. 1989;170(Suppl):2-6; discussion 16-9.

5. Boucher AL, Pereira B, Decousus S, Goutte M, Goutorbe F, Dubois A, et al. Endoscopy-based management decreases the risk of postoperative recurrences in Crohn's disease. World J Gastroenterol. 2016 Jun 7;22(21):5068-78. doi: 10.3748/wjg.v22.i21.5068.

6. American Gastroenterological Association medi-

\section{Адрес для корреспонденции}

220116, Республика Беларусь,

г. Минск, пр. Дзержинского, д. 83,

кафедра детской хирургии,

тел. моб.: +375 029 32-902-32,

e-mail: anvoron@mail.ru,

Воронецкий Александр Николаевич

\section{Сведения об авторах}

Воронецкий А.Н., к.м.н., доцент кафедры детской хирургии УО «Белорусский государственный медицинский университет».

Поступила 18.05.2016 г.

Принята в печать 28.08.2016 г. cal position statement: Guidelines for the evaluation and management of chronic diarrhea. 1999 Jun;116(Is 6):1461-63. doi: http://dx.doi.org/10.1016/S00165085(99)70512-3.

7. Proekt klinicheskikh rekomendatsii po diagnostike i lecheniiu vzroslykh patsientov s bolezn'iu Krona [Project clinical guidelines for the diagnosis and treatment of adult patients with Crohn's disease]. Koloproktologiia. Prilozhenie 2013;(3,45): 22-38

8. Santos-Antunes J, Cardoso H, Lopes S, Marques M, Nunes AC, Macedo G. Capsule enteroscopy is useful for the therapeutic management of Crohn's disease. World J Gastroenterol. 2015 Nov 28;21(44):12660-66. doi: $10.3748 /$ wjg.v21.i44.12660.

Published online 2015 Nov 28. doi: 10.3748/wjg.v21. i44.12660 PMCID: PMC4658621

9. Kilcoyne A1, Kaplan JL1, Gee MS1. Inflammatory bowel disease imaging: Current practice and future directions. World J Gastroenterol. 2016 Jan 21;22(3):91732. doi: $10.3748 /$ wjg.v22.i3.917.

10. Bennett JL, Ha CY, Efron JE, Gearhart SL, Lazarev MG, Wick EC. Optimizing perioperative Crohn's disease management: role of coordinated medical and surgical care. World J Gastroenterol. 2015 Jan 28;21(4):1182-88. doi: 10.3748/wjg.v21.i4.1182.

11. Bettenworth D, Nowacki TM, Cordes F, Buerke B, Lenze F. Assessment of stricturing Crohn's disease: Current clinical practice and future avenues. World $J$ Gastroenterol. 2016 Jan 21;22(3):1008-16. doi: 10.3748/ wjg.v22.i3.1008.

Published online 2016 Jan 21. doi: 10.3748/wjg.v22. i3.1008 PMCID: PMC4716016

12. Podugu A, Tandon K, Castro FJ. Crohn's disease presenting as acute gastrointestinal hemorrhage. World J Gastroenterol. 2016 Apr 28;22(16):4073-78. doi: 10.3748/wjg.v22.i16.4073

Published online 2016 Apr 28. doi: 10.3748/wjg.v22. i16.4073 PMCID: PMC4837426

13. Voronetskii AN, Liakh OM, Dergachev AV. Primenenie endovideokapsuly dlia diagnostiki patologii tonkoi kishki u detei [Application of endovideocapsule for diagnosing pathology of the small intestine in children]. Zdravookhranenie. 2015;(5):32-34.

14. Voronetskii AN, Liakh OM, Leskovskaia SV, Savanovich II, Dergachev AV. Primenenie rastvorimoi kapsuly dlia diagnostiki prokhodimosti tonkoi kishki $\mathrm{u}$ detei [The use of soluble capsules to diagnose intestinal permeability in children]. Zdravookhranenie. 2016;(4):28-32.

\section{Address for correspondence}

220116, the Republic of Belarus,

Minsk, Dzerzhinsky Ave., 83,

Department of pediatric surgery.

Tel.: +375 029 32-902-32

E-mail: anvoron@mail.ru

Alexander N. Voronetsky

\section{Information about the authors}

Voronetsky A.N. PhD, Ass. Professor of department of the pediatric surgery, EE «Belarusian State Medical University».

Received 18.05.2016

Accepted 28.08.2016 